\title{
Development of a Joint Prediction Model Based on Both the Radiomics and Clinical Factors for Predicting the Tumor Response to Neoadjuvant Chemoradiotherapy in Patients with Locally Advanced Rectal Cancer
}

This article was published in the following Dove Press journal: Cancer Management and Research

\author{
Yang Liu $\mathbb{D}^{1, *}$ \\ Feng-Jiao Zhang ${ }^{2, *}$ \\ Xi-Xi Zhao ${ }^{3, *}$ \\ Yuan Yang ${ }^{4}$ \\ Chun-Yi Liang ${ }^{3}$ \\ Li-Li Feng ${ }^{5}$ \\ Xiang-Bo Wan $\left(\mathbb{D}^{5}\right.$ \\ Yi Ding' \\ Yao-Wei Zhang' \\ 'Department of Radiation Oncology, \\ Nanfang Hospital, Southern Medical \\ University, Guangzhou, Guangdong, \\ 5I05I5, People's Republic of China; \\ ${ }^{2}$ Shanghai Concord Medical Cancer \\ Center, Shanghai, 20000I, People's \\ Republic of China; ${ }^{3}$ Medical Imaging \\ Center, Nanfang Hospital, Southern \\ Medical University, Guangzhou, \\ Guangdong, 510515, People's Republic of \\ China; ${ }^{4}$ Guangdong Provincial Key \\ Laboratory of Medical Image Processing, \\ School of Biomedical Engineering, \\ Southern Medical University, Guangzhou, \\ Guangdong, 510515, People's Republic of \\ China; ${ }^{5}$ Department of Radiation \\ Oncology, The Sixth Affiliated Hospital, \\ Sun Yat-Sen University, Guangzhou, \\ Guangdong, 51 0655, People's Republic of \\ China
}

*These authors contributed equally to this work

Correspondence: Yi Ding; Yao-Wei Zhang Tel +8620-62786616

Email dingyil97980@।26.com;

weiyaozhang2@163.com
Purpose: Neoadjuvant chemoradiotherapy (nCRT) has become the standard treatment for locally advanced rectal cancer (LARC). However, the accuracy of traditional clinical indicators in predicting tumor response is poor. Recently, radiomics based on magnetic resonance imaging (MRI) has been regarded as a promising noninvasive assessment method. The present study was conducted to develop a model to predict the pathological response by analyzing the quantitative features of MRI and clinical risk factors, which might predict the therapeutic effects in patients with LARC as accurately as possible before treatment.

Patients and Methods: A total of 82 patients with LARC were enrolled as the training cohort and internal validation cohort. The pre-CRT MRI after pretreatment was acquired to extract texture features, which was finally selected through the minimum redundancy maximum relevance (mRMR) algorithm. A support vector machine (SVM) was used as a classifier to classify different tumor responses. A joint radiomics model combined with clinical risk factors was then developed and evaluated by receiver operating characteristic (ROC) curves. External validation was performed with 107 patients from another center to evaluate the applicability of the model. Results: Twenty top image texture features were extracted from 6192 extracted-radiomic features. The radiomics model based on high-spatial-resolution T2-weighted imaging (HR-T2WI) and contrast-enhanced T1-weighted imaging $(\mathrm{T} 1+\mathrm{C})$ demonstrated an area under the curve (AUC) of 0.8910 (0.8114-0.9706) and 0.8938 (0.8084-0.9792), respectively. The AUC value rose to 0.9371 (0.8751-0.9997) and 0.9113 (0.8449-0.9776), respectively, when the circumferential resection margin (CRM) and carbohydrate antigen 19-9 (CA19-9) levels were incorporated. Clinical usefulness was confirmed in an external validation cohort as well (AUC, 0.6413 and 0.6818).

Conclusion: Our study indicated that the joint radiomics prediction model combined with clinical risk factors showed good predictive ability regarding the treatment response of tumors as accurately as possible before treatment.

Keywords: rectal cancer, neoadjuvant chemoradiotherapy, magnetic resonance imaging, tumor response

\section{Introduction}

The incidence and mortality of colorectal cancer rank the third in the world. ${ }^{1}$ Furthermore, the incidence rate of rectal cancer is increasing, accounting for $30 \%$ of the total incidence rate of colorectal cancer. ${ }^{2,3}$ Therefore, the treatment of locally 
advanced rectal cancer (LARC) has been drawn more attention. Neoadjuvant chemoradiotherapy (nCRT) before total mesorectal excision (TME), which is of great positive value to control the progress of the disease, has been confirmed to significantly increase the tumor downgrade rate. ${ }^{4}$ Approximately $20 \%$ of patients show a pathological complete response (pCR), and they have better diseasefree survival (DFS) after nCRT. ${ }^{5,6}$ Therefor, nCRT has become the standard treatment and has been written into the guidelines earlier. ${ }^{7-9}$ However, recent studies found that patients with a good response (GR), who achieved a tumor regression grade (TRG) of 0 or 1 other than pCR also had a much better prognosis than those with a poor response (PR) after nCRT. ${ }^{10,11}$ Some researchers began to question the use of TME for these patients because of its severe long-term complications and low quality of life, especially permanent colostomy.

Therefore, a lot of studies have been conducted to try to predict the therapeutic effect of neoadjuvant therapy. Molecular biomarkers based on tissue and blood samples have certain predictive value, but invasive operations, which may be unnecessary and result in hysteresis, are required to get specimens. ${ }^{12}$ Noninvasive imaging techniques before surgery are now available to evaluate individualized treatment. Both fluorodeoxyglucose-positron emission tomography (FDG-PET), computed tomography (CT), and magnetic resonance imaging (MRI) have certain diagnostic value, but recent studies have proved that neither FDG-PET nor CT can be used to distinguish therapeutic responses of patients. ${ }^{13}$ MRI has the characteristics of no radiation and high soft tissue resolution, which can clearly identify the structure of each layer of the rectal wall of the patient and has been widely used in the diagnosis of rectal cancer. ${ }^{14,15}$ MRI is commonly used to evaluate patients for a clinical complete response (cCR) because of its accuracy in distinguishing the structure of tissues. ${ }^{16,17}$ Patients with cCR were selected to implement the "organ preservation strategy", which was known as "watch and wait", to avoid unnecessary surgery. ${ }^{18-20}$ However, imaging diagnoses are often influenced by the equipment and doctors. ${ }^{21}$ Additionally, the complexity of the tumor biology itself has been neglected. In recent years, the research on radiomics, which acquire data from medical data and analyze tumor information to estimate treatment response or predict prognosis has attracted much attention and shown great potential for predicting tumor response accurately across different types of cancer. $^{22}$ With the development of computer technology and image processing software, a great amount of objective and quantitative texture feature data can be extracted based on texture feature analysis of images, which has the advantages of being noninvasive, quantitative, and easy to obtain and can be followed up dynamically. ${ }^{23}$ Moreover, these data are closely associated with the biological information of different tumors. ${ }^{24,25}$ For instance, it has been widely reported that MRI radiomics features might be used as potential imaging biomarkers for early prediction of LARC response to neoadjuvant treatment. ${ }^{26-32}$ Although MRI radiomics feature is more informative than traditional imaging, it is not possible to evaluate the tumor biological characteristics of patients only from the perspective of radiomics owing to the heterogeneity and clinical complexity of tumors.

In this present study, we tried to develop a multimodal prediction model by analyzing the quantitative characteristics of multi-sequence MRI using the method of radiomics combined with clinical features to predict the pathological response of LARC to nCRT. We hoped to explore the feasibility of using noninvasive methods to predict the therapeutic effects in patients with LARC as accurately as possible before treatment.

\section{Patients and Methods}

\section{Patients}

This analysis was approved by the Nanfang Hospital Institutional Review Board and the Institutional Review Board of the Sixth Affiliated Hospital, Sun Yat-sen University. The informed consent requirement was waved because the study caused no extra physical, financial or psychosocial harm to any patient and had no influence on the treatment strategies. We guaranteed that the patient data would be used only for this study and not for any other purposes, and adhered to the Declaration of Helsinki. Two independent cohorts of the patients with LARC who underwent nCRT from two different medical centers were enrolled in the study. The inclusion criteria were as follows: (1) clinical II or III stage rectal cancer, (2) histologically confirmed adenocarcinoma, (3) intensitymodulated radiation therapy (IMRT) including 50 Gy delivered for 25 fractions and received standard chemotherapy including capecitabine, oxaliplatin plus capecitabine or oxaliplatin plus 5-fluorouracil during radiotherapy, (4) high-resolution MRI data and clinical risk factors within 1-2 weeks before nCRT, and (5) TME performed within 6-8 weeks after radiotherapy. The 
exclusion criterion was a history of malignancy or inflammatory bowel disease. From December 2012 to December 2017, a total of 189 patients were enrolled in the present study from the two independent medical centers. The training cohort and internal validation cohort that comprised 82 patients obtained from Nanfang Hospital and the external validation cohort that comprised 107 patients obtained from the Sixth Affiliated Hospital, Sun Yat-sen University, all fulfilling the same enrollment criteria. Baseline epidemiological and clinical factors were obtained from medical records. The patient selection process is summarized in Figure 1.

\section{MRI Acquisition and Analysis}

Patients in our hospital were scanned with 3.0-T MR (Achieva; Philips) while others underwent 1.5-T MRI (Optima 360; GE Medical Systems) using a phased-array coil in the cooperating institution. Bowels were prepared with anisodamine to slow down intestinal peristalsis in both institutions. MRI was conducted 1-2 weeks before the start of chemoradiation, including T2-weighted imaging (T2WI), high-spatial-resolution T2WI, T1WI, contrast-enhanced T1WI $(\mathrm{T} 1+\mathrm{C})$ and diffusion-weighted imaging (DWI) MRI. All MRI parameters in the study are summarized in Table 1 . Owing to the missing sequences or poor image quality, only HR-T2WI and T1 $+\mathrm{C}$ were included in this study while others were excluded. Each image was evaluated by two independent professional radiologists with more than 10 years of experience in imaging diagnosis of rectal cancer. The location and size of the tumor as well as the distance from the tumor to the anal canal were recorded. Other clinical risk factors of primary tumor such as circumferential resection margin (CRM), extramural vascular invasion (EMVI) and tumor circumferential ratio were also confirmed.

\section{Scoring the Pathological Response}

Tumor regression grade (TRG), as described in the 8th edition of the American Joint Committee on Cancer (AJCC), was used to score the pathological responses to nCRT in the study. The number of tumor cells and the degree of fibrosis were evaluated by using this scoring system with scores ranging from 0 to 3 . TRG 0 (no viable cancer cells) is equivalent to $\mathrm{pCR}$, while TRG 3 (minimal tumor regression and little fibrosis) means poor or no response. Experienced gastrointestinal pathologists who did not know the MRI data of these patients analyzed tumor sections stained with hematoxylin and eosin in both institutions. Finally, the rectal patients were divided into two groups by the therapeutic effect: patients with TRG $0-1$ were assigned to the good response (GR) group, and patients with TRG 2-3 were assigned to the poor response (PR) group.

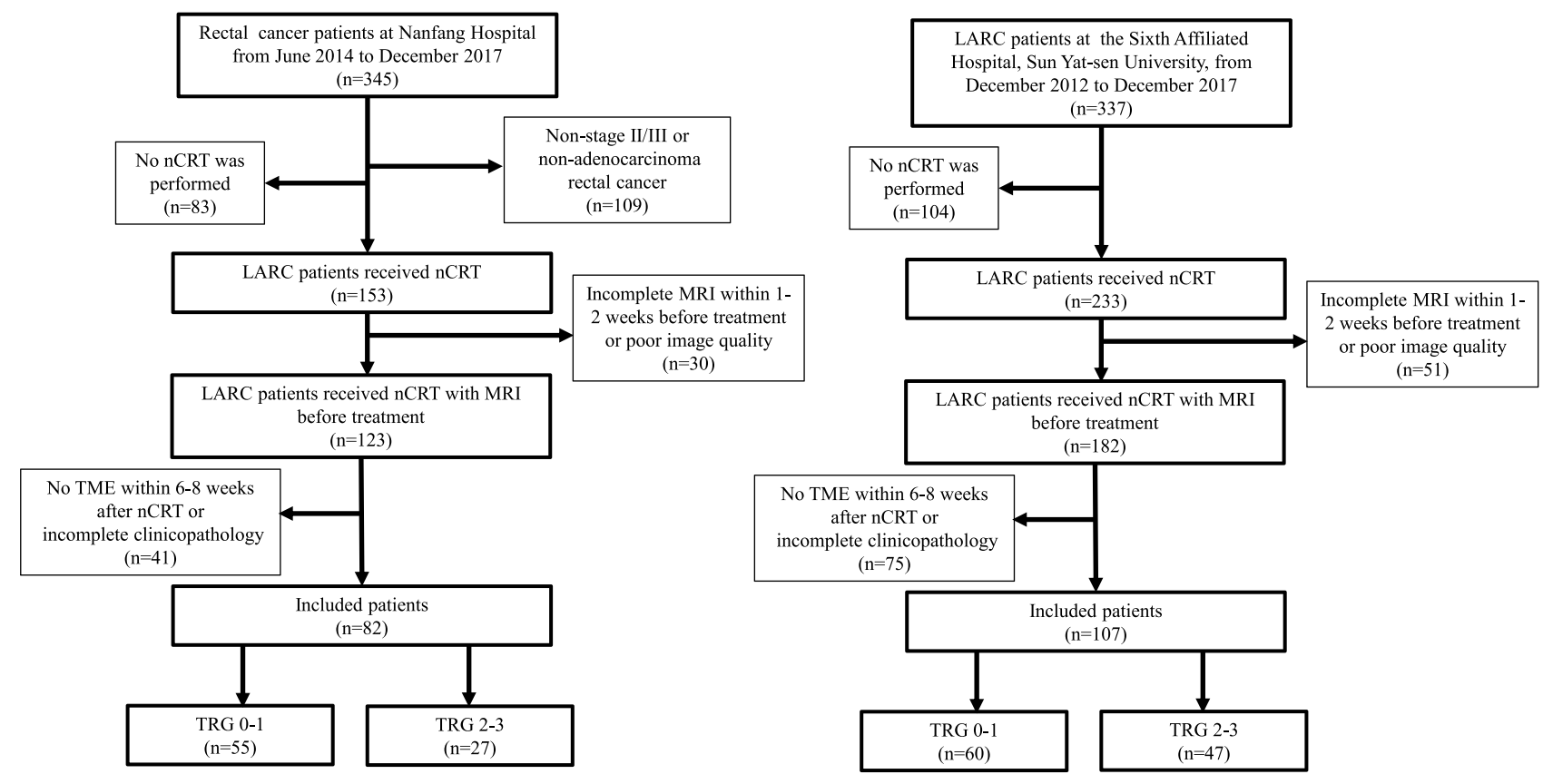

Figure I Flowchart. 
Table I MRI Parameters

\begin{tabular}{|l|c|c|c|c|c|}
\hline \multirow{2}{*}{ MRI Parameters } & \multicolumn{3}{|c|}{ T2-Weighted Image } & Contrast-Enhanced TI-Weighted Imaging \\
(TI+C)
\end{tabular}

\section{Extraction of Texture Features}

The texture features were selected automatically with the commercial ITK-SNAP software used by two experienced radiologists who have more than 2 years of experience in texture feature extraction. Tumors, instead of the entire rectal area as in other studies, were outlined and segmented as a regions of interest (ROI) layer-by-layer on the HR-T2WI and $\mathrm{T} 1+\mathrm{C}$ while using all other image sequences as references. The sample of ROI sample is shown in Figure 2. The volume of interest (VOI) could be acquired from the ROI using three-dimensional volume reconstruction. We used $3 \mathrm{D}$ Slicer software was used to remove the offset field of all the input images in our hospital. The gray value of all images was unified to 256 by normalization, while the form of all images was standardized through three-dimensional transformation. MATLAB software was used to program the extracted image features, which can be generally classified into five categories: gabor texture, gray-level co-occurrence matrix, gray-level run-length matrix, gray-level size zone matrix and neighborhood gray-tone difference matrix. The four parameters of feature extraction such as Ratios, Scale, $\mathrm{Ng}$, and Quant.algo were selected to assist the extraction of radiomic texture features. This procedure was conducted for any combination of feature extraction parameters. Therefore, 144 different corresponding experiments combined with 43 characteristics were required. Finally, 6192 texture features $(43 \times 144)$ were obtained.

The minimum redundancy maximum relevance (mRMR) algorithm was applied to select features from the 6192 extracted radiomic features. The mRMR aims to maximize the correlation between features and categorical variables while minimizing the correlation between different features. It is a measure based on mutual information, which evaluates the degree of correlation between features and target attributes, regardless of the distribution of data. Formally, the mutual information of two discrete random variables $\mathrm{X}$ and $\mathrm{Y}$ can be defined as:

$$
I(X ; Y)=\sum_{y \in Y} \sum_{x \in X} p(x, y) \log \left(\frac{p(x, y)}{p(x) p(y)}\right)
$$

For continuous random variables, the mutual information of $\mathrm{X}$ and $\mathrm{Y}$ can be rewritten as:

$$
I(X ; Y)=\int_{Y} \int_{X} p(x, y) \log \left(\frac{p(x, y)}{p(x) p(y)}\right) d x d y
$$

Feature variables that have the maximum relevance with the target category and the minimum redundancy with the maximum mutual information value were repeatedly selected. In our study, 300 features were selected from 


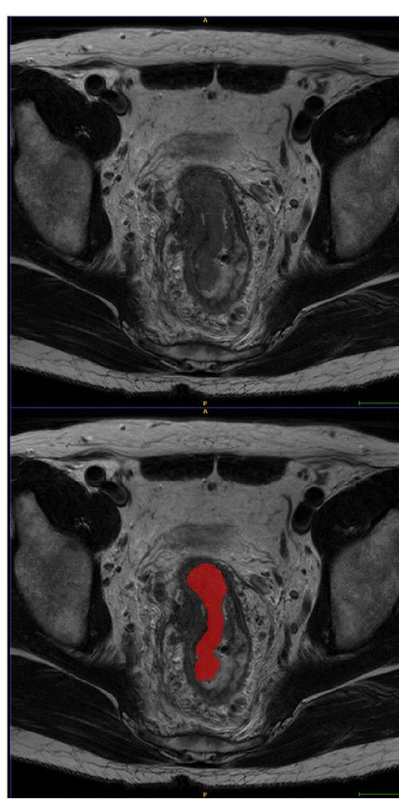

A

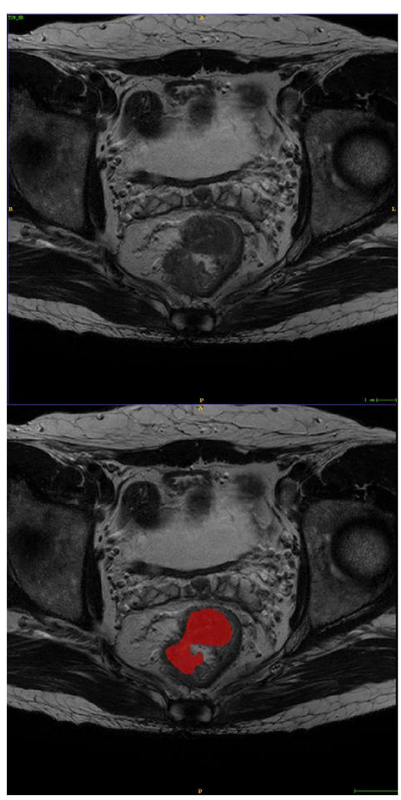

E

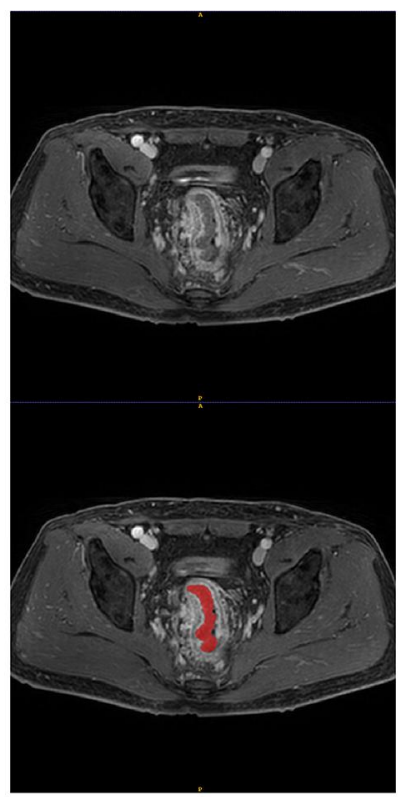

B

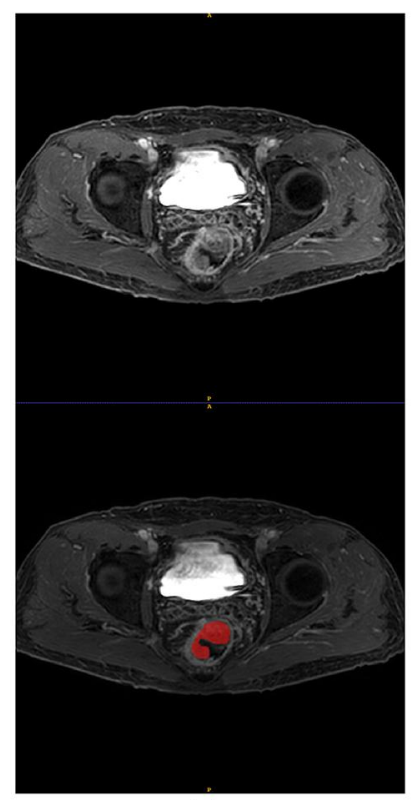

$\mathbf{F}$

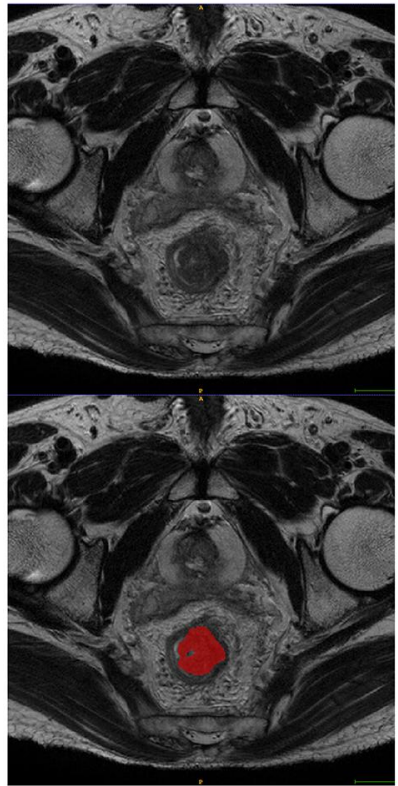

C

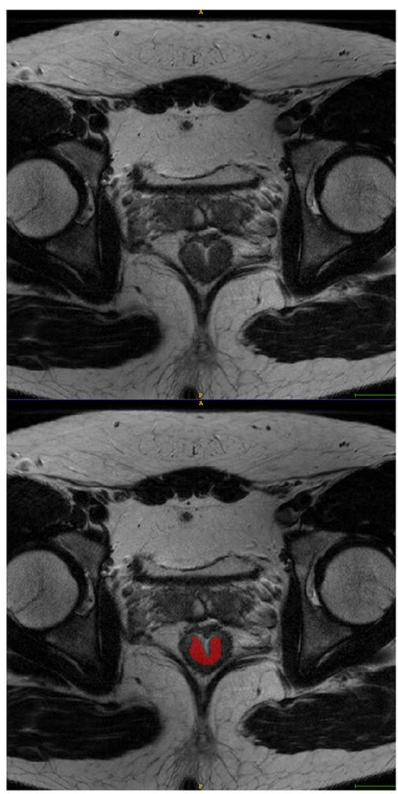

G

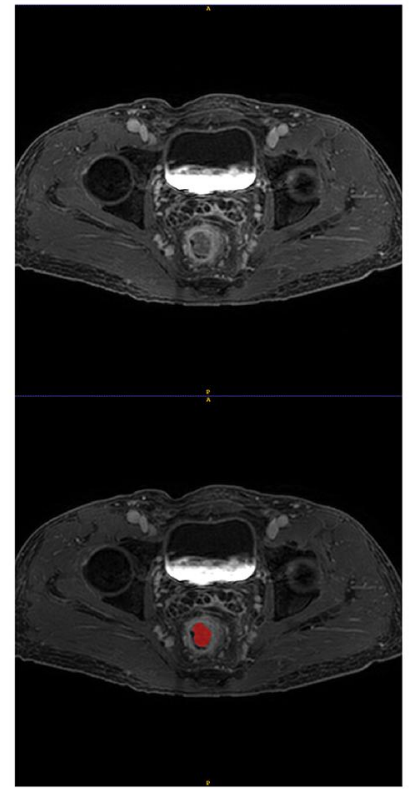

D

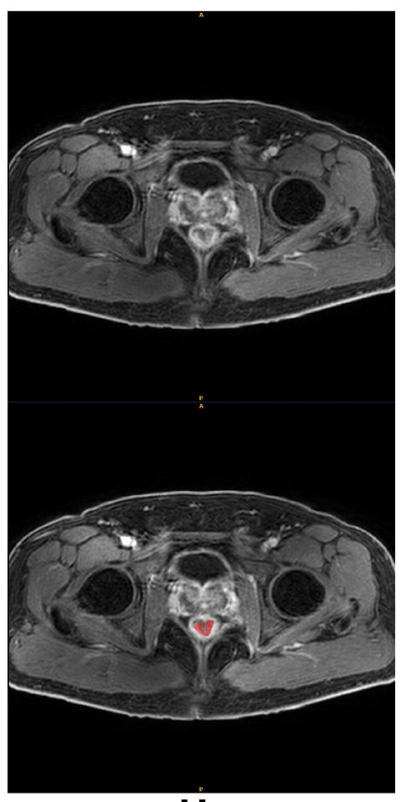

H

Figure 2 Pretreatment MRI and ROI of four male patients with rectal cancer at stage of cT4aN2bM0, who was (A and B) TRG 0, (C and D) TRG I, (E and F) TRG 2 and ( $\mathbf{G}$ and $\mathbf{H})$ TRG 3 after TME, respectively.

6192 texture features in the training set through the mRMR algorithm.

The random forest algorithm was used on the training data to assess variable importance with classification trees. We chose classification and regression trees as the basic unit of the random forest. A random sample with replacement was taken to analyze each decision tree by adopting bootstrap sampling in the input training set samples. With continuously repeated sampling, the probability that the sample will not be picked in $\mathrm{m}$ times is $(1-1 / \mathrm{m}) \mathrm{m}$. Approximately $36.8 \%$ of the samples in the initial dataset were never present in the sampled dataset:

$$
\lim _{m \rightarrow \infty}\left(1-\frac{1}{m}\right)^{m} \rightarrow \frac{1}{e} \approx 0.368
$$

In this paper, the selection of each feature was changed to compare the corresponding prediction error. The larger the prediction error is, the more important the features are. 
These features have a greater impact on the prediction result. Therefore, the importance of each feature can be ranked. Finally, we selected the top 20 image texture features of the training set by prioritizing and repeated the texture feature extraction steps to obtain the texture feature values under different MRI sequences.

\section{Selection of Clinical Factors}

Individual variables were analyzed for significant differences using the $t$-test and the chi-square test for differently distributed parameters. Group results were reported as mean or frequency. A two-sided $\mathrm{P}<0.05$ was considered statistically significant to discriminate GR from PR. We evaluated patients' demographic factors such as sex, age, height, and weight as well as the diameter, location, circumferential ratio and stage of tumors. CRM, EMVI and tumor markers including carcinoembryonic antigen (CEA), carbohydrate antigen (CA)19-9, and CA72-4 were also considered. Based on the statistical analysis, CRM, CA19-9 and CA72-4 were incorporated into the joint model as clinical risk factors.

\section{Development and Validation of Model}

Many redundant features were eliminated in the previous feature selection process. The forward search method was used in our study and the optimal feature subsets were selected in order to avoid combinatorial explosion. We performed the forward search to select the optimal feature subset. A support vector machine (SVM) was used as a classifier to classify and predict two different therapeutic effects. Based on the training set, this method finds a partition hyperplane in the sample space and separates the samples of different categories. The hyperplane can be divided by the following linear equation:

$$
\omega^{T} x+b=0
$$

The training sample points closest to the hyperplane are called "support vectors". The SVM aims to find partitioned hyperplanes with "maximum spacing":

$$
\begin{gathered}
\max _{\omega, b} \frac{2}{\|\omega\|} \\
\text { s.t. } y_{i}\left(\omega^{T} x+b\right) \geq 1, i=1,2, \ldots, m .
\end{gathered}
$$

Multiple models of radiomics features with or without clinical features in different MR sequences were established to classify and predict different therapeutic effects, using the SVM as a classifier.

Finally, we built the model with two sequences, respectively, of HR-T2WI and T1+C. The 10-fold crossvalidation was used for internal validation with the training set data because of the limited cases allotted, whereas the data from the cooperating institution were used for external validation. The differential clinical factors were then incorporated into the combined model to predict the response of nCRT for LARC and repeat the previous validation. Predictive ability was compared using the receiver operating characteristic (ROC) curve.

\section{Results}

\section{Patients Characteristics}

According to the inclusion and exclusion criteria, a total of 82 of the 345 patients who underwent nCRT in our hospital were enrolled as the training set and internal validation set and 107 of 337 patients in the cooperating institution were finally enrolled as the external validation set. The percentage of patients that achieved TRG 0 was approximately $22.0 \%(18 / 82)$ in our hospital and approximately $21.4 \%(22 / 103)$ in the cooperating hospital. The baseline data of patients are summarized in Table 2. In the training and the internal validation cohort, there were 55 patients $(67.1 \%)$ with GR and 27 patients (32.9\%) with PR. There were no significant differences between the two groups in baseline characteristics, such as sex, age, height and weight, etc. No significant differences were also observed in terms of tumor location, clinical stage, and pathological features, which were not associated with the response to nCRT. It is worth noting that circumferential resection margin (CRM), which was evaluated by MRI, was significantly associated with the clinical outcomes of patients. The positive ratio of CRM was lower in the GR group than in the PR group ( $38 \%$ vs $67 \%, \mathrm{P}=0.015$ ). Regarding laboratory tests, two (CA19-9 and CA72-4) of the three gastrointestinal cancer tumor markers showed statistically significant differences between the two groups, suggesting that the level of CA19-9 and CA72-4 before treatment may affect the predictors of treatment response. Based on this analysis, CRM, CA19-9 and CA72-4 were considered to be incorporated into the joint model as clinical risk factors.

\section{Development and Evaluation of Radiomics Model}

Finally, 20 texture feature values from 6192 features were selected. The SVM was used as a classification method to predict GR or PR in different MRI sequences. The ROC curves used to compare the predictive 
Table 2 Clinical Characteristics of Patients

\begin{tabular}{|c|c|c|c|c|c|c|}
\hline \multirow[t]{2}{*}{ Characteristics } & \multicolumn{3}{|c|}{ Training and Internal Validation Cohort } & \multicolumn{3}{|c|}{ External Validation } \\
\hline & $\begin{array}{c}\text { Good Response } \\
\qquad(n=55)\end{array}$ & $\begin{array}{l}\text { Poor Response } \\
\qquad(n=27)\end{array}$ & $p$ value & $\begin{array}{c}\text { Good Response } \\
\qquad(n=60)\end{array}$ & $\begin{array}{l}\text { Poor Response } \\
\qquad(n=47)\end{array}$ & $p$ value \\
\hline Sex & & & 0.764 & & & 0.565 \\
\hline Male & 39 (70.9\%) & 20 (74.1\%) & & $43(71.7 \%)$ & $36(76.6 \%)$ & \\
\hline Female & $16(29.1 \%)$ & 7 (25.9\%) & & $17(28.3 \%)$ & II (23.4\%) & \\
\hline Age & $53.62 \pm 9.82$ & $54.44 \pm 9.55$ & 0.719 & $53.22 \pm 10.70$ & $57.25 \pm 11.78$ & 0.067 \\
\hline Height (cm) & $165.65 \pm 6.43$ & $165.07 \pm 7.79$ & 0.721 & $164.75 \pm 7.86$ & $165.36 \pm 7.11$ & 0.685 \\
\hline Weight (kg) & $62.12 \pm 9.61$ & $62.82 \pm 10.42$ & 0.765 & $63.62 \pm 10.21$ & $62.82 \pm 8.99$ & 0.589 \\
\hline Tumor diameter (cm) & $4.53 \pm 1.62$ & $4.46 \pm 1.12$ & 0.814 & $4.91 \pm 1.97$ & $4.64 \pm 1.37$ & 0.373 \\
\hline Tumor location & & & 0.834 & & & 0.168 \\
\hline Low & $28(50.9 \%)$ & 15 (55.6\%) & & $37(61.7 \%)$ & $21(44.7 \%)$ & \\
\hline Middle & $22(40.0 \%)$ & 9 (33.3\%) & & $21(35.0 \%)$ & $25(53.2 \%)$ & \\
\hline High & $5(9.1 \%)$ & $3(11.1 \%)$ & & $2(3.3 \%)$ & I (2.1\%) & \\
\hline $\begin{array}{l}\text { Tumor circumferential } \\
\text { ratio }\end{array}$ & & & 0.542 & & & \\
\hline 0-35\% & $2(3.6 \%)$ & $0(0 \%)$ & & NA & NA & \\
\hline $35-70 \%$ & $15(27.3 \%)$ & 9 (33.3\%) & & NA & NA & \\
\hline $70-100 \%$ & 38 (69.1\%) & $18(66.7 \%)$ & & NA & NA & \\
\hline CRM & & & $0.015^{*}$ & & & $0.035^{*}$ \\
\hline Positive & 21 (38.2\%) & $18(66.7 \%)$ & & $26(43.3 \%)$ & $30(63.8 \%)$ & \\
\hline Negative & $34(61.8 \%)$ & $9(33.3 \%)$ & & $34(56.7 \%)$ & $17(36.2 \%)$ & \\
\hline EMVI & & & 0.222 & & & \\
\hline Positive & $24(43.6 \%)$ & $8(29.6 \%)$ & & NA & NA & \\
\hline Negative & 31 (56.4\%) & 19 (70.4\%) & & NA & NA & \\
\hline Clinical tumor stage & & & 0.505 & & & 0.396 \\
\hline cTI & $\mathrm{I}(\mathrm{I} .8 \%)$ & $0(0 \%)$ & & $0(0 \%)$ & $0(0 \%)$ & \\
\hline cT2 & $2(3.6 \%)$ & $3(11.1 \%)$ & & I (I.7\%) & $2(4.3 \%)$ & \\
\hline cT3 & $22(40 \%)$ & $12(44.4 \%)$ & & $42(70.0 \%)$ & $32(68.1 \%)$ & \\
\hline cT4a & 21 (38.2\%) & $10(37.0 \%)$ & & 7 (I I.7\%) & 9 (19.1\%) & \\
\hline cT4b & $9(16.4 \%)$ & $2(7.4 \%)$ & & $10(16.7 \%)$ & $4(8.5 \%)$ & \\
\hline Clinical nodal stage & & & 0.287 & & & 0.168 \\
\hline cNO & $0(0 \%)$ & I (3.7\%) & & 7 (1 I.7\%) & $8(17.0 \%)$ & \\
\hline $\mathrm{cNI}$ & $8(14.5 \%)$ & $8(29.6 \%)$ & & $19(31.7 \%)$ & $21(44.7 \%)$ & \\
\hline cN2 & 47 (85.5\%) & $18(66.7 \%)$ & & 34 (56.7\%) & 18 (38.3\%) & \\
\hline Tumor differentiation & & & 0.181 & & & $0.48 I$ \\
\hline Well & $18(32.7 \%)$ & 7 (25.9\%) & & $14(23.3 \%)$ & $13(27.7 \%)$ & \\
\hline Moderate & $35(63.6 \%)$ & $16(59.3 \%)$ & & 38 (63.3\%) & $31(66.0 \%)$ & \\
\hline Poor & $2(3.6 \%)$ & $4(14.8 \%)$ & & $8(13.3 \%)$ & $3(6.4 \%)$ & \\
\hline \multicolumn{7}{|l|}{ Tumor marker } \\
\hline CEA & $3.6 \mathrm{I}(\mathrm{I} .49 \sim 10.0 \mathrm{I})$ & $5.84(2.14 \sim 20.64)$ & 0.069 & $2.96(1.75 \sim 6.46)$ & $4.79(2.49 \sim 8.49)$ & $0.037^{*}$ \\
\hline CAI9-9 & $12.23(6.77 \sim 16.28)$ & 22.1 (I6.4 29.97) & $0.000 * * *$ & 5.35 (2.30 9.57) & $23.06(20.00 \sim 30.50)$ & $0.000^{* * *}$ \\
\hline CA72-4 & $2.38(1.25 \sim 7.40)$ & 10.29 (3.08 17.45) & $0.00 I^{* *}$ & NA & NA & \\
\hline
\end{tabular}

(Continued) 
Table 2 (Continued).

\begin{tabular}{|c|c|c|c|c|c|c|}
\hline \multirow[t]{2}{*}{ Characteristics } & \multicolumn{3}{|c|}{ Training and Internal Validation Cohort } & \multicolumn{3}{|c|}{ External Validation } \\
\hline & $\begin{array}{c}\text { Good Response } \\
\qquad(n=55)\end{array}$ & $\begin{array}{l}\text { Poor Response } \\
\quad(n=27)\end{array}$ & $p$ value & $\begin{array}{l}\text { Good Response } \\
\qquad(n=60)\end{array}$ & $\begin{array}{c}\text { Poor Response } \\
\quad(n=47)\end{array}$ & $p$ value \\
\hline $\begin{array}{l}\text { Vessel invasive } \\
\text { Positive } \\
\text { Negative }\end{array}$ & $\begin{array}{c}3(5.6 \%) \\
52(94.5 \%)\end{array}$ & $\begin{array}{c}2(7.4 \%) \\
25(92.6 \%)\end{array}$ & 0.728 & $\begin{array}{c}0(0 \%) \\
60(100 \%)\end{array}$ & $\begin{array}{c}2(4.3 \%) \\
45(95.7 \%)\end{array}$ & 0.191 \\
\hline $\begin{array}{l}\text { Neural invasion } \\
\text { Positive } \\
\text { Negative }\end{array}$ & $\begin{array}{c}2(3.6 \%) \\
53(96.3 \%)\end{array}$ & $\begin{array}{c}3(11.1 \%) \\
24(88.9 \%)\end{array}$ & 0.184 & $\begin{array}{c}2(3.3 \%) \\
58(96.7 \%)\end{array}$ & $\begin{array}{c}5(10.6 \%) \\
42(89.4 \%)\end{array}$ & 0.236 \\
\hline
\end{tabular}

Notes: $* \mathrm{P}<0.05 ; * * \mathrm{P}<0.01$; $* * * \mathrm{P}<0.001$.

capacity of the two groups are displayed in Figure 3A. The HR-T2WI radiomics model including 20 texture features had an internally verified the area under the curve (AUC) value of 0.8910 with a sensitivity of
0.8148 and specificity of 0.7925 . The AUC was 0.5413 in the external validation cohort with a sensitivity of 0.5312 and specificity of 0.5 . The AUC value of the T1 $+\mathrm{C}$ radiomics model including 16 texture features was
A

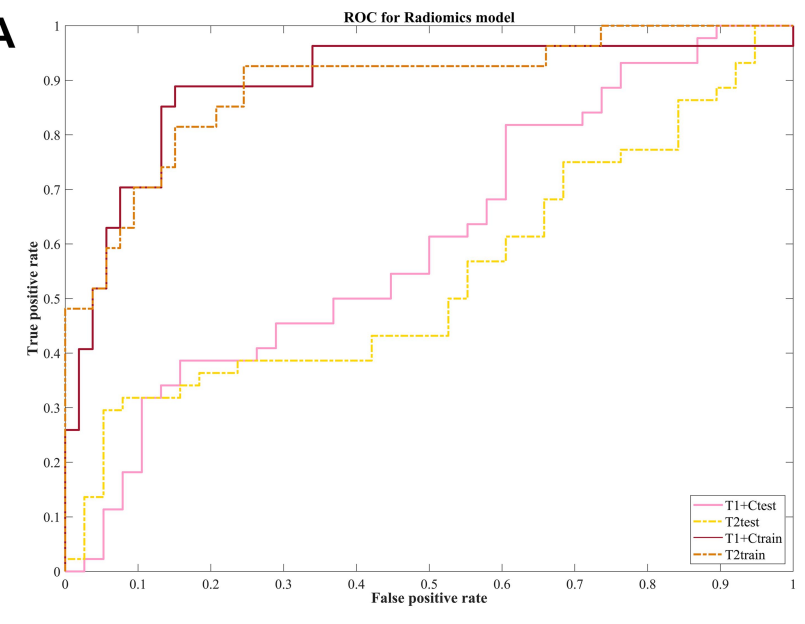

B

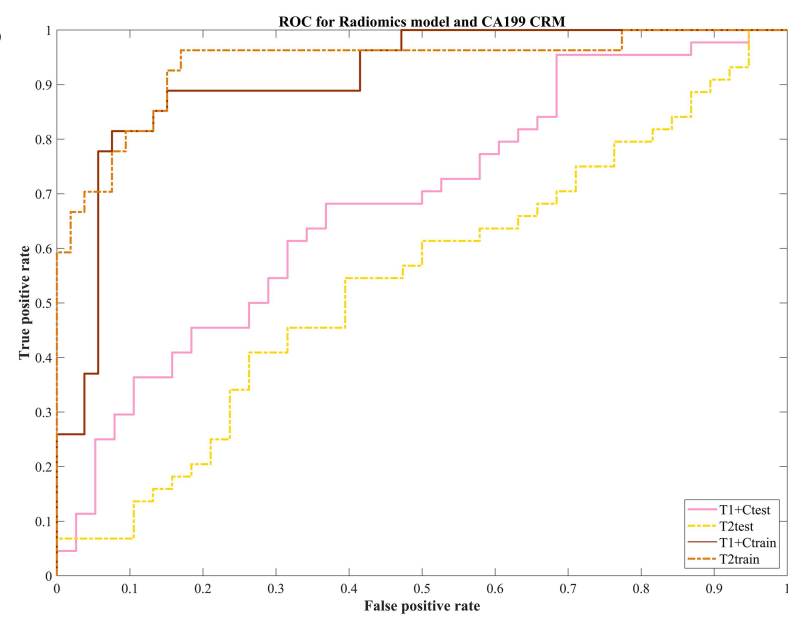

\section{C}

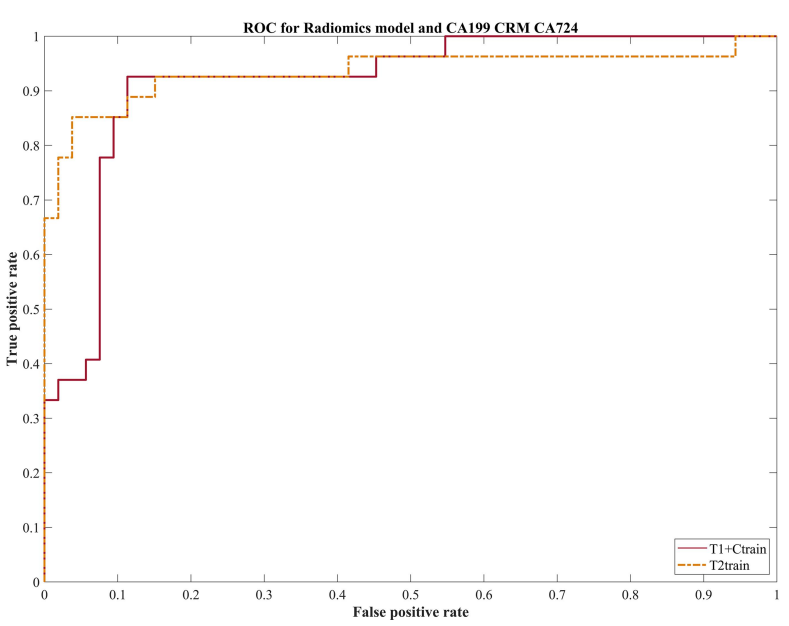

Figure 3 Comparison of ROC curves between internal and external validation of two sequences in (A) radiomic model, (B) joint model and (C) joint model incorporated CA $72-4$. 


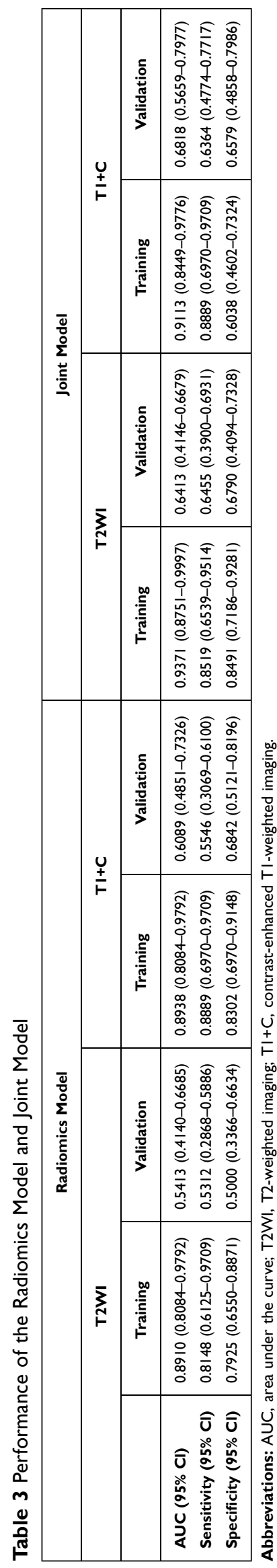

0.8938 with a sensitivity of 0.8889 and specificity of 0.8302. In the external validation cohort, the AUC was 0.6089 with a sensitivity of 0.5546 and specificity of 0.6842 (Table 3).

\section{Combination of Radiomics and Clinical Characteristics}

The clinical features of CRM, CA19-9 and CA72-4 were incorporated to develop the joint model based on radiomics. Because patients from the cooperating institution had no data of CA72-4, we designed two different united models, with and without CA72-4. It is noteworthy that, in the internal validation cohort, the AUC value of the HRT2WI radiomics joint model based on 20 texture features rose to 0.9371 while that of $\mathrm{T} 1+\mathrm{C}$ based on 19 texture features rose to 0.9113 after adding the two clinical features of CA19-9 and CRM. In the external validation cohort, the AUC rose to 0.6413 and 0.6818 , respectively. Finally, we added CRM, CA19-9 and CA72-4 to our joint model and performed internal validation in our hospital. The AUC value of the HR-T2WI radiomics model reached 0.9350 and the value of $\mathrm{T} 1+\mathrm{C} 0.9168$. The ROC curves are displayed in Figure 3B and C. The details of the AUC values, sensitivity and specificity are shown in Table 3.

\section{Discussion}

In the present study, we developed a joint prediction model based on radiomics and clinical factors to predict the possible tumor response to nCRT in patients with LARC. It is worth mentioning that we only outlined the tumor as an ROI, excluding the intestinal lumen. SVM was used as a classifier to distinguish different therapeutic effects. The idea behind classification using such an algorithm was to maximize the spacing between the sample and the decision surface. It is suitable for small samples as well as the nonlinear classification. The results show that radiomics models could predict the therapeutic effect of nCRT in both HR-T2WI and T1+C sequences, and exhibited enhanced prognostic performance when combined with clinical factors, which has been externally validated.

Early imaging studies were used to predict the early efficacy of nCRT for rectal cancer by assessing the rate of volume reduction or the maximum depth of extramural invasion in functional MRI. ${ }^{14,32}$ However, functional MRI is still unable to excavate fully and quantitatively the large amount of information contained in the images. With the development of artificial intelligence algorithms, 
especially the significant progress of deep learning, the birth of radiomics provides more ways to develop models to improve the accuracy of diagnosis, prognosis and prediction. ${ }^{33}$ Recently, radiomics based on MRI was applied in the field of rectal cancer research in an endless stream. Nie et al reported that the role of radiomics is greater than that of traditional imaging indicators through a systematic analysis of multiparametric MRI features. The AUC based on radiomics of multiparametric MRI could be improved to $0.71-0.79$ in predicting pCR instead of 0.54-0.73 using the traditional method. ${ }^{29}$ Subsequently, Liu et al developed and validated a radiomics model for evaluating pCR to nCRT in 222 patients with LARC. The individualized radiomics model was a combination of the radiomics signature and tumor length and showed good discrimination using pre- and posttreatment MRI data based on T2WI and DWI. ${ }^{31}$ Horvat et al also agreed that radiomics based on T2WI showed better classification performance for diagnosing pCR than qualitative assessment. The diagnostic performance was significantly higher using radiomics than T2WI and DWI. ${ }^{27}$ Overall, subsequent studies have also shown that the radiomics models have excellent predictive capability in patients with LARC after CRT. ${ }^{28,34,35}$

Our study is basically consistent with the conclusions of the other studies mentioned above, but there were some obvious advantages. Firstly, our radiomic features were extracted from two sequence separately, and not a single sequence analysis. Rich and realistic radiomics information was obtained from HR-MRI. HR-T2WI has become a highly valued sequence for clinicians owing to its good tissue resolution, which displays the invasion of the rectal wall and surrounding tissue better. ${ }^{36} \mathrm{~T} 1+\mathrm{C}$ sequences, which can be used to indicate tumor blood supply, can be better evaluate tumor invasiveness because the growth, development and metastasis of tumors depend on complex microvessels for nutrition. ${ }^{37}$ Both $\mathrm{T} 2 \mathrm{WI}$ and $\mathrm{T} 1+\mathrm{C}$ sequences showed high predictive value for treatment response in the present study, which is important for predicting the outcome of treatment. Secondly, our study differed from earlier studies limited to radiomics in that clinical data were also incorporated into the model as important factors. CRM refers to the shortest distance between the deepest tumor invasion and the boundary of mesentery resection. ${ }^{38}$ When the distance is less than $1 \mathrm{~mm}, \mathrm{CRM}$ is considered positive, which can be determined accurately by MRI. ${ }^{39}$ The size and degree of invasion of the primary tumor could be assessed by determining whether CRM is involved, which is also closely related to the prognosis of patients. ${ }^{40}$ Meanwhile, tumor markers such as CEA, CA19-9 and CA72-4 are also commonly used for rectal cancer diagnosis and follow-up. A single prognostic markers are often not accurate enough because of the complex biological characteristics of tumors. ${ }^{41}$ In the present study, we demonstrated that positive CRM as well as a high level of CA19-9 and CA72-4 were associated with a worse therapeutic response. Thus, the predictive efficacy of the model was further improved due to the incorporation of clinical factors. Additionally, both the radiomics models and the joint models including clinical data were externally validated to confirm their clinical usefulness, which might provide the possibility for a multicenter study.

In summary, the present study developed a joint prediction model based on radiomics features as well as clinically risk factors to accurately predict the possible response of tumors to nCRT for LARC. Unfortunately, there are still some limitations in our study. We divided the patients into two groups, GR (TRG $0-1$ ) and PR (TRG 2-3), to evaluate the therapeutic effect of nCRT. TRG 0 might be more suitable targets of the predictive model because of the better long-term prognosis. However, the number of patients with TRG 0 was small in our retrospective study. Thus, there may be selection bias with respect to radiomics features and clinical factors. We would like to do further analysis on the patients with TRG 0 in the future. Additionally, some factors such as CEA might be related to tumor response, and the lack of statistically significant differences between the two groups in this study might be due to the small sample size. Thus, we did not incorporate these factors into our model. Lastly, information differences in the images derived from different MRI equipment and parameters of the two centers should be considered. The issue of how to acquire standardized images also needs to be resolved in future. Therefore, the prospective studies in multiple centers are needed for further evaluation and setting up a reliable database of radiomics.

\section{Conclusion}

Despite the limitations, our joint multimodal prediction model showed an improvement in the predictive capability by including clinical risk factors. It is believed that physicians will be able to achieve "risk stratification" of patients based on the analysis of radiomics texture features as well as clinical risk factors of tumors, in the foreseeable future. Our study provides 
more evidences for noninvasive methods to predict the treatment response to tumors, which will be helpful for determining the individualized treatment of patients with LARC.

\section{Acknowledgments}

This work was supported by the National Natural Science Foundation of China (No. 81872470, 81803063 and 81903125), Natural Science Foundation of Guangdong Province, China (No. 2018030310297 and 2018A030310513), Guangdong Medical Research Foundation (No. A2020563) and Southern Hospital Director Foundation (No. 2019B009).

\section{Disclosure}

The authors report no conflicts of interest in this work.

\section{References}

1. Siegel RL, Miller KD, Jemal A, et al. Cancer statistics, 2020. $C A$ Cancer J Clin. 2020;70(1):7-30. doi:10.3322/caac.21590

2. Siegel RL, Miller D, Fedewa SA, et al. Trends in colon and rectal cancer incidence in Australia from 1982 to 2014: analysis of data on over 375,000 cases. Cancer Epidemiol Biomarkers Prev. 2019;28 (1):83-90. doi:10.1158/1055-9965.EPI-18-0523

3. Bailey CE, Hu CY, You YN, et al. Increasing disparities in the agerelated incidences of colon and rectal cancers in the United States, 1975-2010. JAMA Surg. 2015;150(1):17-22. doi:10.1001/jamasurg. 2014.1756

4. Li Y, Wang J, Ma X, et al. A review of neoadjuvant chemoradiotherapy for locally advanced rectal cancer. Int J Biol Sci. 2016;12 (8):1022-1031. doi:10.7150/ijbs. 15438

5. Maas M, Nelemans PJ, Valentini V, et al. Long-term outcome in patients with a pathological complete response after chemoradiation for rectal cancer: a pooled analysis of individual patient data. Lancet Oncol. 2010;11(9):835-844. doi:10.1016/S1470-2045(10)70172-8

6. Smith JJ, Garcia-Aguilar J. Advances and challenges in treatment of locally advanced rectal cancer. $J$ Clin Oncol. 2015;33 (16):1797-1808. doi:10.1200/JCO.2014.60.1054

7. Sanghera P, Wong DW, McConkey CC, et al. Chemoradiotherapy for rectal cancer: an updated analysis of factors affecting pathological response. Clin Oncol. 2008;20(2):176-183. doi:10.1016/j.clon.2007. 11.013

8. Bosset JF, Collette L, Calais G, et al. Chemotherapy with preoperative radiotherapy in rectal cancer. $N$ Engl $J$ Med. 2006;355 (11):1114-1123. doi:10.1056/NEJMoa060829

9. van Gijn W, Marijnen CA, Nagtegaal ID, et al. Preoperative radiotherapy combined with total mesorectal excision for resectable rectal cancer: 12-year follow-up of the multicentre, randomised controlled TME trial. Lancet Oncol. 2011;12(6):575-582. doi:10.1016/S14702045(11)70097-3

10. Dhadda AS, Dickinson P, Zaitoun AM, et al. Prognostic importance Mandard tumour regression grade following pre-operative chemoradiotherapy locally advanced rectal cancer. Cancer. 2011;47 (8):1138-1145

11. Suarez J, Vera R, Balen E, et al. Pathologic response assessed by Mandard grade is a better prognostic factor than down staging for disease-free survival after preoperative radiochemotherapy for advanced rectal cancer. Colorectal Dis. 2008;10(6):563-568. doi:10.1111/j.1463-1318.2007.01424.x
12. Dayde D, Tanaka I, Jain R, et al. Predictive and prognostic molecular biomarkers for response to neoadjuvant chemoradiation in rectal cancer. Int J Mol Sci. 2017;18(3):573. doi:10.3390/ijms18030573

13. Guillem JG, Ruby JA, Leibold T, et al. Neither FDG-PET Nor CT can distinguish between a pathological complete response and an incomplete response after neoadjuvant chemoradiation in locally advanced rectal cancer: a prospective study. Ann Surg. 2013;258 (2):289-295. doi:10.1097/SLA.0b013e318277b625

14. Horvat N, Carlos Tavares Rocha C, Clemente Oliveira B, et al. MRI of rectal cancer: tumor staging, imaging techniques, and management. Radiographics. 2019;39(2):367-387. doi:10.1148/ rg. 2019180114

15. Jhaveri KS, Hosseini-Nik H. MRI of rectal cancer: an overview and update on recent advances. AJR Am J Roentgenol. 2015;205(1):W4255. doi:10.2214/AJR.14.14201

16. Gollub MJ, Blazic I, Felder S, et al. Value of adding dynamic contrast-enhanced MRI visual assessment to conventional MRI and clinical assessment in the diagnosis of complete tumour response to chemoradiotherapy for rectal cancer. Eur Radiol. 2019;29 (3):1104-1113. doi:10.1007/s00330-018-5719-1

17. Martens MH, Maas M, Heijnen LA, et al. Long-term outcome of an organ preservation program after neoadjuvant treatment for rectal cancer. $J$ Natl Cancer Inst. 2016;108(12):djw171. doi:10.1093/jnci/djw171

18. Maas M, Beets-Tan RG, Lambregts DM, et al. Wait-and-see policy for clinical complete responders after chemoradiation for rectal cancer. J Clin Oncol. 2011;29(35):4633-4640. doi:10.1200/JCO.2011.37.7176

19. van der Valk MJM, Hilling DE, Bastiaannet E, et al. Long-term outcomes of clinical complete responders after neoadjuvant treatment for rectal cancer in the International Watch \& Wait Database (IWWD): an international multicentre registry study. Lancet. 2018;391(10139):2537-2545. doi:10.1016/S0140-6736(18)31078-X

20. Smith JJ, Strombom P, Chow OS, et al. Assessment of a Watch-andWait strategy for rectal cancer in patients with a complete response after neoadjuvant therapy. JAMA Oncol. 2019;5(4):e185896. doi:10.1001/jamaoncol.2018.5896

21. Ryan JE, Warrier SK, Lynch AC, et al. Predicting pathological complete response to neoadjuvant chemoradiotherapy in locally advanced rectal cancer: a systematic review. Colorectal Dis. 2016;18(3):234-246. doi:10.1111/codi.13207

22. Aerts HJ, Velazquez ER, Leijenaar RT, et al. Decoding tumour phenotype by noninvasive imaging using a quantitative radiomics approach. Nat. Commun. 2014;5(1):4006. doi:10.1038/ncomms5006

23. Lambin P, Leijenaar RTH, Deist TM, et al. Radiomics: the bridge between medical imaging and personalized medicine. Nat Rev Clin Oncol. 2017;14(12):749-762. doi:10.1038/nrclinonc.2017.141

24. Thawani R, McLane M, Beig N, et al. Radiomics and radiogenomics in lung cancer: a review for the clinician. Lung Cancer. 2018;115:34-41. doi:10.1016/j.lungcan.2017.10.015

25. Valdora F, Houssami N, Rossi F, et al. Rapid review: radiomics and breast cancer. Breast Cancer Res Treat. 2018;169(2):217-229. doi:10.1007/s10549-018-4675-4

26. Huang YQ, Liang CH, He L, et al. Development and validation of a radiomics nomogram for preoperative prediction of lymph node metastasis in colorectal cancer. $J$ Clin Oncol. 2016;34 (18):2157-2264. doi:10.1200/JCO.2015.65.9128

27. Horvat N, Veeraraghavan $\mathrm{H}$, Khan M, et al. MR imaging of rectal cancer: radiomics analysis to assess treatment response after neoadjuvant therapy. Radiology. 2018;287(3):833-843. doi:10.1148/ radiol.2018172300

28. Yi X, Pei Q, Zhang Y, et al. MRI-based radiomics predicts tumor response to neoadjuvant chemoradiotherapy in locally advanced rectal cancer. Front Oncol. 2019;9:552. doi:10.3389/fonc.2019.00552

29. Nie K, Shi L, Chen Q, et al. Rectal cancer: assessment of neoadjuvant chemoradiation outcome based on radiomics of multiparametric MRI. Clin Cancer Res. 2016;22(21):5256-5264. doi:10.1158/1078-0432. CCR-15-2997 
30. Alvarez-Jimenez C, Antunes JT, Talasila N, et al. Radiomic texture and shape descriptors of the rectal environment on post-chemoradiation T2-weighted MRI are associated with pathologic tumor stage regression in rectal cancers: a Retrospective, Multi-Institution Study. Cancers (Basel). 2020;12(8):2027. doi:10.3390/cancers12082027

31. Liu Z, Zhang XY, Shi YJ, et al. Radiomics analysis for evaluation of pathological complete response to neoadjuvant chemoradiotherapy in locally advanced rectal cancer. Clin Cancer Res. 2017;23(23):7253-7262. doi:10.1158/1078-0432.CCR-171038

32. Kalisz KR, Enzerra MD, Paspulati RM. MRI evaluation of the response of rectal cancer to neoadjuvant chemoradiation therapy. Radiographics. 2019;39(2):538-556. doi:10.1148/rg.20 19180075

33. Hosny A, Parmar C, Quackenbush J, et al. Artificial intelligence in radiology. Nat Rev Cancer. 2018;18(8):500-510. doi:10.1038/ s41568-018-0016-5

34. Bulens P, Couwenberg A, Intven $M$, et al. Predicting the tumor response to chemoradiotherapy for rectal cancer: model development and external validation using MRI radiomics. Radiother Oncol. 2020;142:246-252. doi:10.1016/j.radonc.2019. 07.033

35. Cui Y, Yang X, Shi Z, et al. Radiomics analysis of multiparametric MRI for prediction of pathological complete response to neoadjuvant chemoradiotherapy in locally advanced rectal cancer. Eur Radiol. 2019;29(3):1211-1220. doi:10.1007/s00330-0185683-9
36. Pham TT, Liney G, Wong K, et al. Study protocol: multi-parametric magnetic resonance imaging for therapeutic response prediction in rectal cancer. BMC Cancer. 2017;17(1):465. doi:10.1186/s12885017-3449-4

37. Armbruster M, D'Anastasi M, Holzner V, et al. Improved detection of a tumorous involvement of the mesorectal fascia and locoregional lymph nodes in locally advanced rectal cancer using DCE-MRI. Int J Colorectal Dis. 2018;33(7):901-909. doi:10.1007/s00384-018-3083-x

38. Iannicelli E, Di Renzo S, Ferri M, et al. Accuracy of high-resolution MRI with lumen distention in rectal cancer staging and circumferential margin involvement prediction. Korean J Radiol. 2014;15 (1):37-44. doi:10.3348/kjr.2014.15.1.37

39. Kang BM, Park YK, Park SJ, et al. Does circumferential tumor location affect the circumferential resection margin status in mid and low rectal cancer? Asian J Surg. 2018;41(3):257-263. doi:10. 1016/j.asjsur.2016.12.001

40. van der Paardt MP, Zagers MB, Beets-Tan RG, et al. Patients who undergo preoperative chemoradiotherapy for locally advanced rectal cancer restaged by using diagnostic MR imaging: a systematic review and meta-analysis. Radiology. 2013;269(1):101-112. doi:10.1148/ radiol. 13122833

41. Lech G, Słotwiński R, Słodkowski M, et al. Colorectal cancer tumour markers and biomarkers: recent therapeutic advances. World J Gastroenterol. 2016;22(5):1745-1755. doi:10.3748/wjg.v22.i5.1745

\section{Publish your work in this journal}

Cancer Management and Research is an international, peer-reviewed open access journal focusing on cancer research and the optimal use of preventative and integrated treatment interventions to achieve improved outcomes, enhanced survival and quality of life for the cancer patient.
The manuscript management system is completely online and includes a very quick and fair peer-review system, which is all easy to use. Visit http://www.dovepress.com/testimonials.php to read real quotes from published authors. 